\title{
Prediction of Lymph Node Metastasis in Colon Cancer via Platelet to Lymphocyte Ratio and Platelet Count
}

\author{
Oguz Catal, Bahri Ozer and Mustafa Sit \\ Department of General Surgery, Abant Izzet Baysal University Hospital, Bolu, Turkey
}

\begin{abstract}
Objective: To investigate the value of preoperative blood tests in predicting lymph node metastasis in colon cancer patients undergoing surgery.

Study Design: Obervational study.

Place and Duration of Study: General Surgery Department, Bolu Abant Izzet Baysal University Medicine School, Turkey, between December 2012 and December 2018.

Methodology: In 171 colon cancer patients, preoperative inflammatory markers such as albumin, C-reactive protein (CRP), platelet, neutrophil-to-lymphocyte ratio (NLR), and platelet-lymphocyte ratio (PLR) counts were determined.

Results: The present study albumin, CRP and NLR values were also not significantly different between the groups. PLR $(p=0.008)$ and PLT $(p=0.039)$ were significantly different between the lymph node groups. ROC analysis was performed for PLT and PLR for the prediction of lymph node metastasis (Group 2 and 3). Accuracy for PLT was 0.623 and accuracy for PLR was 0.65 . A PLT value $>321.5$ was found to be $63.7 \%$ sensitive and $68.6 \%$ specific in predicting lymph node metastasis. A PLR value $>155.81$ was $70 \%$ sensitive and $41 \%$ specific for predicting lymph node metastasis.

Conclusion: The platelet counts and PLR values are important in predicting lymph node metastasis in colon cancer, preoperatively. Platelet and PLR values may be more specific and sensitive in predicting preoperative lymph node metastasis in colon cancer patients.
\end{abstract}

Key Words: Colon cancer, Platelet, Platelet to lymphocyt ratio (PLR), Lymph node, Metastasis, Markers of inflammation.

How to cite this article: Catal O, Ozer B, Sit M. Prediction of lymph node metastasis in colon cancer via platelet to lymphocyte ratio and platelet count. J Coll Physicians Surg Pak 2020; 30(3):250-253.

\section{INTRODUCTION}

Colon cancer is the third most common cancer type in the world. The most important prognostic variable in colon cancer is lymph node metastases. The number of lymph nodes with metastases is adversely associated with life expectancy. Survival for 5 years is around $60 \%$ when there is a single lymph node metastasis; $35 \%$ if there are 2-5 lymph nodes metastasis, and $20 \%$ if there are $\geq 6$ lymph nodes metastasis.

In the 19th Century, Virchow described a link between cancer and inflammation and suggested that lymphocytic infiltrates in the areas of chronic inflammation may reflect the origin of cancer. ${ }^{1}$ Chronic inflammation has been documented in different types of cancer.2,3 There are also inflammatory responses in the microenvironment of most neoplastic tissues that do not involve in any linkage to the inflammatory process. ${ }^{3}$

Correspondence to: Dr. Oguz Catal, Department of Surgery, Abant Izzet Baysal University Hospital, Golkoy, 14280, Bolu, Turkey

E-mail: otuzogur@gmail.com

Received: October 16, 2019; Revised: January 17, 2020;

Accepted: January 28, 2020
Different biochemical or hematological markers have been used to measure the effect of systemic inflammatory responses in cancer patients including high C-reactive protein (CRP), neutrophils and platelets and hypo-albuminemia. Among these, platelet/ lymphocyte ratio (PLR), ${ }^{4}$ and neutrophil / lymphocyte ratio (NLR) 5 were prominent.

PLR has been found to provide prognostic information in various types of cancer. Preoperative PLR can be a clinically significant factor for assessment of prognosis of resectable colorectal cancer.6 Preoperative PLR values have been reported to be associated with reccurrence period in patients undergoing curative surgical resection in stage 2 and 3 colon cancer. ${ }^{7}$ To authors' knowledge, association of preoperative PLR values with lymph node metastasis has not been documented previously.

The aim of this study was to investigate the value of preoperative PLR via platelet count in predicting lymph node metastasis in colon cancer patients undergoing surgery.

\section{METHODOLOGY}

A total of 171 patients, who were operated for colon cancer between December 2012 and December 2018 at 
the General Surgery Clinic of Bolu Abant Izzet Baysal University were evaluated, retrospectively. Preoperative hemogram, biochemistry and postoperative pathology reports were assessed. Preoperative albumin, CRP, platelet (PLT) counts were determined. Neutrophillymphocyte ratio (NLR) was calculated by dividing neutrophil count to lymphocyte count. Finally, platelet / lymphocyte ratio (PLR) was determined by division of platelet count to lymphocyte count.

Using TNM classification of AJCC (Nx: Regional lymph nodes cannot be evaluated; N0: No local lymph node metastasis; N1: 1-3 metastasis in the pericolic lymph node; N2: Metastasis in 4 or more pericolytic lymph nodes; N3: Metastasis in any lymph node at a main vessel level), patients were divided into three groups according to the lymph node metastasis. There were no patients with $\mathrm{Nx}$ and N3 classifications. Therefore, Group 1 included patients with N0 classification, Group 2 included patients with N1, and Group 3 included patients with N2. Patients taking neoadjuvant radiotherapy, having rectal involvement or liver metastasis or undergoing emergency operation due to ileus or perforation were excluded.

Variables with normally distribution were analysed using One-way ANOVA and Tukey HSD tests. Normality of variables was checked with Shapiro-Wilk test. Kruskal-

Table I: Classification of the patients.

\begin{tabular}{ll}
\hline Gender & $56 \%$ \\
Male $(n=96)$ & $44 \%$ \\
Female $(n=75)$ & $16.3 \%$ \\
\hline Localisation & $16.3 \%$ \\
Caecum ( $n=28)$ & $0.5 \%$ \\
Ascending colon $(n=28)$ & $5.2 \%$ \\
Hepatic flexura $(n=1)$ & $5.2 \%$ \\
Transvers colon $(n=9)$ & $9,3 \%$ \\
Splenic flexura $(n=9)$ & $22.2 \%$ \\
Descending colon $(n=16)$ & $24.5 \%$ \\
Sigmoid colon $(n=38)$ & \\
Rectosigmoid colon $(n=42)$ & $1.75 \%$ \\
\hline T wall invasion & $4.6 \%$ \\
Tis $(n=3)$ & $16.3 \%$ \\
T1 $(n=8)$ & $50.8 \%$ \\
T2 $(n=28)$ & $26.3 \%$ \\
T3 $(n=87)$ & \\
T4 $(n=45)$ & $53.2 \%$ \\
Lymph node involvement & $25.7 \%$ \\
N0 (Group 1) $n=91$ & $21.05 \%$ \\
N1 (Group 2) $n=44$ & \\
N2 (Group 3) $n=36$ &
\end{tabular}

Wallis and Bonferroni adjusted Dunn's tests were used for analysing non-normally distributed continuous variables. Pearson's correlations were calculated for assessing the relationship between the laboratory parameters and lymph node. For determining the optimum cut-off values of statistically significant variables for identifying positive lymph node receiver-operating characteristic (ROC) curve was used. All the analyses were performed with the Statistical Package for Social Sciences 25.0 for Windows (SPSS Inc., Chicago, Illinois, USA) and the results with a level of $p<0.05$ were considered to be significant.

\section{RESULTS}

One hundred and seventy-one colon cancer patients were included in the study. The mean age of the patients was 65.93 (16-89). The characteristics of the patients was shown in Table I.

Age, MPV, albumin, CRP and NLR values were not significantly different between the groups. PLR $(p=0.008)$ and PLT $(p=0.039)$ were significantly different between the groups (Table II). Post-hoc Bonferroni adjusted Dunn's test were applied for pairwise comparisons between the groups. Platelet and PLR were found to be significantly higher in Group 3 than Group $1(p=0.039$ and $\mathrm{p}=0.008$, respectively).

ROC analysis was performed for PLT and PLR for the prediction of lymph node metastasis (Group 2 and 3 ). Accuracy for PLT was 0.623 and accuracy for PLR was

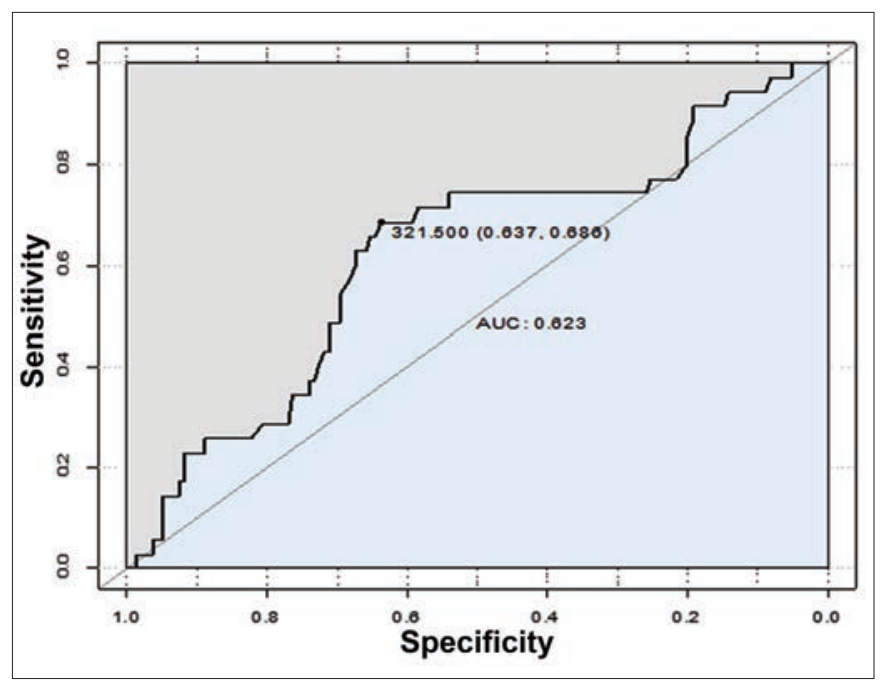

Figure 1: ROC analysis of platelet value.

Table II: Preoperative blood test parameters.

\begin{tabular}{l|l|l|l|l}
\hline Variables & Group 1 $(\mathrm{n}=91)$ & Group 2 $(\mathrm{n}=44)$ & Group 3 $(\mathrm{n}=36)$ & $\mathrm{p}$-value \\
\hline Albumin & $3.79 \pm 0.56$ & $3.79 \pm 0.58$ & $3.58 \pm 0.58$ & 0.162 \\
CRP & $8(0.10-259.43)$ & $4.9(0-256)$ & $8.7(0.1-195.61)$ & 0.224 \\
Platelet & $280(117-746)$ & $281.5(154-876)$ & $343.5(170-627)$ & 0.039 \\
NLR & $3.10(1.13-30.55)$ & $3.06(1.10-18.53)$ & $3.85(1.47-29.88)$ & 0.118 \\
PLR & $167.7(41.79-684.4)$ & $183.9(68.13-494.9)$ & $257.5(86.67-702)$ & 0.008 \\
\hline
\end{tabular}




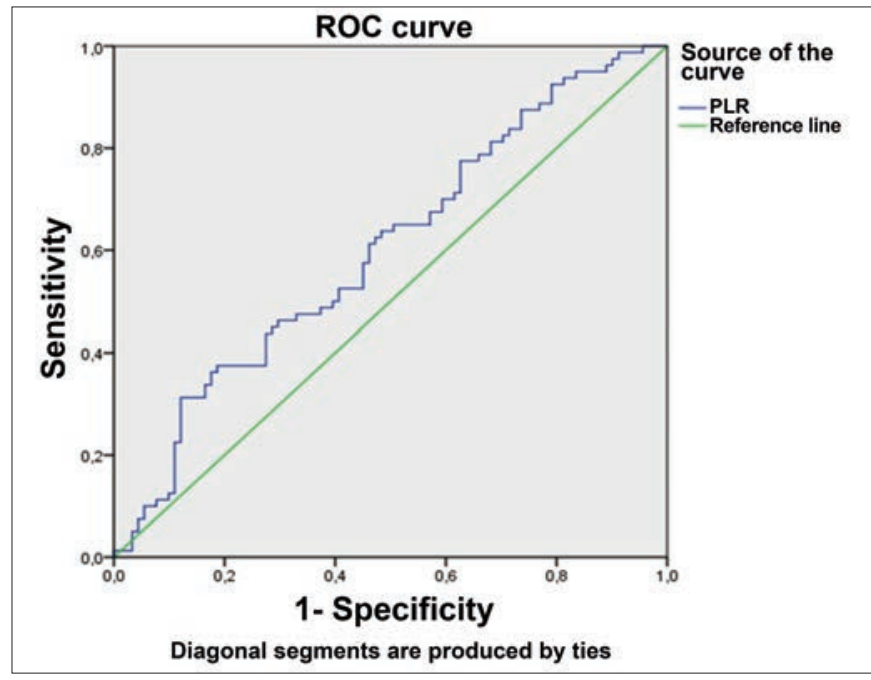

Figure 2: ROC analysis of PLR value.

0.65. A PLT value $>321.5$ was found to be $63.7 \%$ sensitive and $68.6 \%$ specific in predicting lymph node metastasis (Figure 1).

A PLR value $>155.81$ was $70 \%$ sensitive and $41 \%$ specific for predicting lymph node metastasis (Figure 2).

\section{DISCUSSION}

The main finding of present study was that PLR and platelet counts were associated with lymph node metastasis in colon cancer patients. In colon cancer patients, $24-41 \%$ of patients die within 5 years following surgical resection for curative purposes; besides 56$78 \%$ of patients who underwent palliative resection for colon cancer, die within two years. ${ }^{8}$ It is important to predict lymph node metastasis preoperatively because lymph node metastasis is the most important independent factor affecting these rates. The most important tool for preoperative staging of colon cancer is computer tomography (CT).

One of the main limitations of CT is low accuracy in lymph node staging. ${ }^{9}$ The presence of micrometastasis in normal sized lymph nodes and lymph node inflammation-induced growth lead to misdiagnoses in sizedependent evaluation.10 Various studies have been given at various rates on the preoperative prediction of $\mathrm{CT}$ in lymph node metastasis in colon cancer. Cademartiri et al. showed $55 \%$ sensitivity and $65 \%$ specificity for $\mathrm{N}$ staging. ${ }^{11}$ In another study, CT was $84 \%$ sensitive in evaluating lymph node metastasis in colon cancer and $54 \%$ specific. 10

Platelets facilitate proliferation of tumors by detaching tumor from its primary site, and masking the tumor cells from the immune surveillance. Furthermore, platelets help in metastasis; thereby, promoting tumor development to distant body parts. ${ }^{12}$ When the platelet counts in patients with colon cancer were examined, a strong relationship was found between the primary tumor and high platelet count in CRC patients. It is stated that they underline the predictive power of thrombocytosis in CRC patients. ${ }^{13}$ However, as far as the studies are concerned, not a single study has been found suggesting that the number of platelets in colon cancer can predict lymph node metastasis. In the findings of this study, significant result was found between the number of platelets and the number of positive lymph nodes. Platelet count was found to be significant in predicting lymph node metastasis 4 or more. When the ROC analysis was performed, platelet value was found that the prediction of lymph node metastasis was $63.7 \%$, and the specificity was $68.6 \%$ when the platelet count was higher than 321.5 .

Previous studies showed that PLR is associated with prognosis of various types of cancers. ${ }^{14}$ Some publications on the relationship between colon cancer and PLR have shown that PLR is strongly associated with survival time, disease-free survival, and cancer-specific survival in CRC. High PLR may be a promising prognostic biomarker for CRC. 15 In a meta-analysis, it was found that high PLR was an important biomarker for weak overall survival, disase-free survival and recurrencefree survival in patients with CRC. 16 In a study, showing the relationship between PLR and preoperative prediction of lymph node metastasis in colon cancer, they found a significant relationship between PLR and positive lymph node ratio. ${ }^{17}$ In this study, there was a correlation between PLR values and positive lymph node number. ROC analysis showed that PLR value was $70 \%$ sensitive and $41 \%$ specific in predicting preoperative lymph node metastasis in colon cancer above 155.81.

In the light of this information, there was a significant difference between the groups in predicting preoperative lymph node metastasis in colon cancer patients for platelet and PLR values. Platelet and PLR values have similar results with computed tomography and sensitivity and specificity in predicting lymph node metastasis in colon cancer.

Platelet and PLR values may be more specific and sensitive predicting preoperative lymph node metastasis in colon cancer patients. Especially when the number of metastatic lymph nodes is more than 3 , it is more sensitive in prediction.

\section{CONCLUSION}

The platelet counts and PLR values are significantly important in predicting lymph node metastasis in colon cancer, preoperatively. Neoadjuvant chemotherapy can be performed in patients with metastatic lymph node positivity by examining platelet and PLR values. However, this study needs to be supported with larger patient series. 
ETHICAL APPROVAL:

The Ethics Committee of Bolu Abant Izzet Baysal University Clinical Researches approved this study (Number: 414, Date: 28/12/2018).

\section{PATIENTS' CONSENT:}

Necessary permissions were obtained from patients regarding the study.

\section{CONFLICT OF INTEREST:}

Authors declared no conflict of interest.

\section{AUTHORS' CONTRIBUTION:}

OC: Data collection and curation, resources, validation, writing-original draft, writing-review and editing.

BO: Formal analysis, visualisation.

MS: Project administration, visualisation.

\section{REFERENCES}

1. Roxburgh CS, Salmond JM, Horgan PG, Oien KA, McMillan DC Comparison of the prognostic value of inflammation-based pathologic and biochemical criteria in patients undergoing potentially curative resection for colorectal cancer. Ann Surg 2009; 249:788-93.

2. Coussens LM, Werb Z. Inflammation and cancer. Nature 2002; 420:860-7.

3. Colotta F, Allavena P, Sica A, Garlanda C, Mantovani A. Cancer-related inflammation, the seventh hallmark of cancer: links to genetic instability. Carcinogenesis 2009; 30:1073-81.

4. Smith RA, Bosonnet L, Raraty M, Sutton R, Neoptolemos JP, Campbell $\mathrm{F}$, et al. Preoperative platelet-lymphocyte ratio is an independent significant prognostic marker in resected pancreatic ductal adenocarcinoma. Am J Surg 2009; 197:466-72.

5) Walsh S, Cook E, Goulder F, Justin T, Keeling N. Neutrophillymphocyte ratio as a prognostic factor in colorectal cancer. J Surg Oncol 2005; 91:181-4.

6. Liu H, Du X, Sun P, Xiao C, Xu Y, Li R. Preoperative plateletlymphocyte ratio is an independent prognostic factor for resectable colorectal cancer. Nan Fang Yi Ke Da Xue Xue Bao 3; 33:70-3.
7. Szkandera J, Pichler M, Absenger G, Stotz M, Arminger F, Weissmueller $\mathrm{M}$, et al. The elevated preoperative platelet to lymphocyte ratio predicts decreased time to recurrence in colon cancer patients. Am J Surg 2014; 208:210-4.

8. McArdle C, Hole D. Outcome following surgery for colorectal cancer: Analysis by hospital after adjustment for case mix and deprivation. Br J Cancer 2002; 86:331-5.

9. Tudyka V, Blomqvist L, Beets Tan R, Boelens PG, Valentini V, van de Velde CJ, et al. EURECCA consensus conference highlights about colon \& rectal cancer multidisciplinary management: The radiology experts review. Eur J Surg Oncol 2014; 40:469-75.

10. Elibol FD, Obuz F, Sökmen S, Terzi C, Canda AE, Sagol Ö, et al. The role of multidetector CT in local staging and evaluation of retroperitoneal surgical margin involvement in colon cancer. Diagn Interv Radiol 2016; 22:5-12.

11. Cademartiri F, Luccichenti G, Rossi A, Pavone P. Spiral hydro$\mathrm{CT}$ in the evaluation of colo-sigmoideal cancer. Radiol Med 2002; 104:295-306.

12. Asghar S, Parvaiz F, Manzoor S. Multifaceted role of cancer educated platelets in survival of cancer cells. Thromb Res 2019; 177:42-50.

13. Josa V, Krzystanek M, Eklund AC, Salamon F, Zarand A, Szallasi Z, et al. Relationship of postoperative thrombocytosis and survival of patients with colorectal cancer. Int J Surg 2015; 18:1-6.

14. Yu J, Ding Z, Yang Y, Liu S. Increased platelet-to-lymphocytes ratio is associated with poor long-term prognosis in patients with pancreatic cancer after surgery. Medicine (Baltimore) 2018; 97:e11002.

15. Peng HX, Lin K, He BS, Pan YQ, Ying HQ, Hu XX, et al. Platelet to lymphocyte ratio could be a promising prognostic biomarker for survival of colorectal cancer: A systematic review and meta analysis. FEBS Open Bio 2016; 6:742-50.

16. Gu X, Gao XS, Qin S. Elevated platelet to lymphocyte ratio is associated with poor survival outcomes in patients with colorectal cancer. PloS One 2016; 11:e0163523.

17. Lee HY, Choi HJ, Park KJ, Shin JS, Kwon HC, Roh MS, et al. Prognostic significance of metastatic lymph node ratio in nodepositive colon carcinoma. Ann Surg Oncol 2007; 14:1712-7. 